\title{
Efeito da aclimatização em novos usuários de próteses auditivas lineares e não lineares
}

\author{
Acclimatization effect on new users of linear and non linear \\ hearing aids
}

\author{
Tiago Petry ${ }^{1}$, Sinéia Neujahr dos Santos ${ }^{2}$, Maristela Julio Costa ${ }^{3}$
}

\begin{abstract}
RESUMO
Objetivo: Verificar a influência do tempo de uso da amplificação sobre o benefício obtido com as próteses auditivas, para novos usuários e comparar os resultados de usuários de amplificação linear e não linear com compressão dinâmica. Métodos: foram examinados 37 indivíduos portadores de perda auditiva neurossensorial de grau leve a moderadamente severo, novos usuários de próteses auditivas. Os indivíduos foram reunidos em dois grupos: Grupo Linear - composto por 13 indivíduos adaptados com amplificação linear; e Grupo Não Linear - composto por 24 indivíduos adaptados com compressão dinâmica. As avaliações foram realizadas 14 e 90 dias após a adaptação das próteses. Foram obtidos, em campo livre, os limiares de reconhecimento de sentenças no silêncio e no ruído e os índices percentuais de reconhecimento de sentenças no silêncio e no ruído. Os dados foram analisados por meio dos testes de Wilcoxon e Mann-Whitney. Resultados: Os valores obtidos após 14 e 90 dias de uso da amplificação, para ambos os grupos, não apresentaram diferenças significativas. Quando comparados os grupos, os valores também não revelaram diferenças estatísticas. Conclusão: Não foi verificada influência do tempo de uso da amplificação sobre o benefício obtido com as próteses. Não houve diferenças nos resultados alcançados pelos novos usuários de amplificação linear daqueles obtidos pelos novos usuários de amplificação não linear.
\end{abstract}

Descritores: Audição; Perda auditiva neurossensorial; Auxiliares de audição; Audiometria da fala; Testes de discriminação da fala

\section{INTRODUÇÃO}

Um dos recursos terapêuticos para os indivíduos portadores de perda de audição é o uso de próteses auditivas ${ }^{(1)}$. A indicação médica de seleção e adaptação de próteses auditivas deve ocorrer quando a deficiência auditiva não pode ser solucionada com a prescrição de medicamentos ou quando não há indicação cirúrgica.

Os progressos tecnológicos das próteses auditivas visam proporcionar ao portador de perda de audição o restabelecimento da estimulação acústica com melhor qualidade sonora, em especial para os sons da fala ${ }^{(2)}$.

As próteses auditivas processavam grande parte dos sons de entrada de forma linear. Entretanto, há mais de uma déca-

Trabalho realizado no Laboratório de Próteses Auditivas do Serviço de Atendimento Fonoaudiológico da Universidade Federal de Santa Maria - UFSM - Santa Maria (RS), Brasil.

(1) Mestre, Perito Criminal do Instituto Geral de Perícias do Estado de Santa Catarina - IGP - Florianópolis (SC), Brasil.

(2) Mestre, Fonoaudióloga clínica - Santa Maria (RS), Brasil.

(3) Doutor, Professora Adjunto do Departamento de Fonoaudiologia da Universidade Federal de Santa Maria - UFSM - Santa Maria (RS), Brasil.

Endereço para correspondência: Tiago Petry. R. Luiz Alegretti, 66, Centro,

Viadutos (RS), Brasil, CEP: 99820-000. E-mail: tpfono@yahoo.com.br

Recebido em: 22/8/2009; Aceito em: 7/3/2010 da, foram lançados os sistemas que processam os sinais de entrada de forma não linear. Nesse caso, a classificação clínica das próteses auditivas ocorre conforme o processamento do sinal é realizado, principalmente para a fala ${ }^{(3)}$. Quando não há variação do ganho, ou seja, a quantidade de amplificação é a mesma para todas as intensidades sonoras de entrada, as próteses auditivas são lineares; quando há alteração automática nos parâmetros de amplificação, especialmente do ganho, as próteses são classificadas como não lineares ${ }^{(2)}$. No grupo de próteses auditivas não lineares estão incluídas aquelas com compressão dinâmica ou WDRC (Wide Dynamic Range Compression), que costumam fornecer maior ganho para sons mais baixos e menos perceptíveis, enquanto provêem menos ganho para os sons médios e mais intensos ${ }^{(4)}$.

O indivíduo, ao iniciar o uso de próteses auditivas, tem a reintrodução da estimulação sonora por meio da amplificação e ingressa em um período denominado aclimatização perceptual, caracterizado por evoluções no desempenho em testes de fala ao longo do tempo, observadas somente após um intervalo que varia de seis a 12 semanas seguintes à adaptação( ${ }^{(5)}$, decorrentes da capacidade que as vias auditivas centrais têm de se reorganizarem e alterarem sua função em resposta à estimulação auditiva ${ }^{(1)}$. Há relatos de que o fenômeno da aclimatização pode ser influenciado pelas tecnologias distintas das próteses auditivas ${ }^{(6)}$. 
É importante avaliar os benefícios obtidos ao longo do tempo, com o uso de próteses auditivas de diferentes tecnologias e verificar se um tipo de tecnologia pode proporcionar melhores resultados do que outro. Para averiguar essas diferenças no desempenho, é de grande valia a utilização de testes que possibilitem que as avaliações sejam efetuadas em condições que se assemelhem às encontradas no cotidiano, com o uso de frases em detrimento a palavras isoladas e com circunstâncias que simulem situações de silêncio e competição sonora. Um dos materiais que permite ao examinador avaliar o reconhecimento de fala de seu paciente, tanto no silêncio quanto no ruído, é o teste Listas de Sentenças em Português (LSP) ${ }^{(7)}$.

Dessa forma, objetivou-se, por meio do teste LSP, avaliar novos usuários de próteses auditivas com amplificação linear ou amplificação não linear do tipo WDRC, com a finalidade de verificar a influência do tempo de uso da amplificação sobre o benefício obtido com as próteses, e investigar se existem diferenças entre os resultados alcançados pelos usuários de amplificação linear e aqueles obtidos pelos usuários de amplificação não linear.

\section{MÉTODOS}

Esta pesquisa foi realizada no Laboratório de Próteses Auditivas da Universidade Federal de Santa Maria. O estudo é parte integrante do projeto "Pesquisa e Base de Dados em Saúde Auditiva", aprovado pelo Comitê de Ética em Pesquisa com certificado de número 0138.0.243.000-06, e registrado no Gabinete de Projetos da instituição sob o número 019731. Todos os indivíduos participantes da pesquisa, depois de receberem explicações sobre objetivo e metodologia propostos, assinaram o Termo de Consentimento Livre e Esclarecido.

Para que um indivíduo fosse incluído em um dos grupos de estudos, deveria contemplar todos os critérios: ter idade igual ou superior a 18 anos; possuir diagnóstico audiológico de perda auditiva do tipo neurossensorial de grau leve a moderadamente severo ${ }^{(8)}$; adquirida no período pós-lingual; apresentar o Limiar de Reconhecimento de Fala, na melhor orelha, com resultado igual ou inferior a $65 \mathrm{~dB} \mathrm{NA}$; ter a indicação do uso binaural de próteses auditivas; receber adaptação de próteses auditivas com amplificação linear ou amplificação não linear do tipo WDRC; não apresentar nenhum fator que pudesse interferir no teste, como alterações neurológicas e/ou de fluência verbal.

Foram pré-selecionados, de janeiro a outubro de 2008 , indivíduos que compareceram ao Laboratório de Próteses Auditivas para iniciar os procedimentos para concessão de próteses auditivas e que cumpriram as condições de elegibilidade do grupo de estudo mencionado. Dentre os 210 pacientes atendidos, foram pré-selecionados 44 , dos quais 37 concluíram as avaliações e compuseram o referido grupo, após exclusão dos que apresentaram problemas de saúde ou qualquer outra impossibilidade de retorno para a reavaliação.

Os 37 indivíduos foram reunidos de acordo com o tipo de amplificação proporcionado pelas próteses auditivas em dois grupos:

- Grupo Linear: novos usuários de próteses auditivas com amplificação linear;
- Grupo Não Linear: novos usuários de próteses auditivas com compressão do tipo WDRC.

Desse modo, o Grupo Linear foi composto por 13 indivíduos com idades entre 40 e 78 anos (média de idade = 64,23 anos), sendo três do gênero masculino e dez do gênero feminino.

O Grupo Não Linear foi constituído por 24 indivíduos com idades entre 28 e 77 anos (média de idade $=61,13$ anos), sendo 13 do gênero masculino e 11 do gênero feminino.

Não houve, entre os grupos, diferenças relacionadas ao grau da perda auditiva, índice de reconhecimento de fala ou tempo de utilização das próteses auditivas. Ocorreu, entretanto, uma distinção referente ao número de componentes de cada grupo, 13 para amplificação linear e 24 para amplificação não linear.

Para classificar o tipo de amplificação gerada pela prótese auditiva, foi considerado o modo como o ganho é processado. $\mathrm{Na}$ amplificação linear, o ganho da prótese é constante para todas as intensidades de sinal de entrada. Para as próteses auditivas com compressão dinâmica ou WDRC, que processam os sons, incluindo a fala, de forma não linear, o ganho é variável conforme os níveis de pressão sonora de entrada ${ }^{(4)}$.

Todos os indivíduos foram examinados em duas diferentes sessões de avaliação: 14 e 90 dias após a adaptação das próteses auditivas.

Nessas avaliações e reavaliações, efetuadas entre os meses de janeiro de 2008 e janeiro de 2009 , os sujeitos foram submetidos à pesquisa do:

- LRSS - Limiar de reconhecimento de sentenças no silêncio;

- LRSR - Limiar de reconhecimento de sentenças no ruído; - IPRSS - Índice percentual de reconhecimento de sentenças no silêncio;

- IPRSR - Índice percentual de reconhecimento de sentenças no ruído.

Nas duas sessões de avaliação os sujeitos estavam usando suas próteses auditivas nas regulagens estabelecidas pela equipe técnica responsável pelos pacientes do Laboratório de Próteses Auditivas, sem nenhuma modificação entre as sessões de avaliação e com verificação prévia da carga da bateria.

Antes da realização da primeira avaliação, foi efetuada uma anamnese, por meio de um questionário constituído por questões fechadas, as quais forneceram informações referentes a dados pessoais, queixas auditivas, história otológica, hábitos de vida diária e nível de escolaridade dos sujeitos estudados. Também foram anotados, em protocolo padrão, os dados referentes à Audiometria Tonal Liminar, Limiar de Reconhecimento de Fala e Índice Percentual de Reconhecimento de Fala que comprovaram a perda auditiva do sujeito e que serviram de base para a programação das próteses auditivas.

O LRSS, o LRSR, IPRSS e o IPRSR foram obtidos utilizando-se o teste $\operatorname{LSP}^{(7)}$, constituído por uma lista de 25 sentenças ${ }^{(9)}$, sete listas com dez sentenças ${ }^{(10)}$ e um ruído com espectro de fala ${ }^{(11)}$. As sentenças e o ruído estão gravados em $C D$, em canais independentes, e foram apresentados através de um CD player acoplado a um audiômetro. Os resultados foram anotados no protocolo padrão. 
O teste foi aplicado em ambiente acusticamente tratado, em campo livre e com o indivíduo posicionado de frente, a um metro da fonte sonora, a $0^{\circ}-0^{\circ}$ azimute. A sequência de aplicação, tanto para a $1^{\mathrm{a}}$ avaliação quanto para a $2^{\mathrm{a}}$ avaliação, foi:

- apresentação da lista 5B, sem a presença de ruído competitivo e com variação do nível da fala, para obtenção do LRSS;

- apresentação da lista 6B, sem a presença de ruído competitivo e com a fala fixada a $65 \mathrm{~dB}(\mathrm{~A})$, para obtenção do IPRSS;

- apresentação da lista 1B, com a presença de ruído competitivo fixo a $65 \mathrm{~dB}(\mathrm{~A})$ e com variação do nível da fala, para obtenção do LRSR;

- apresentação da lista 2B, com a presença de ruído competitivo e fala fixos, ambos a $65 \mathrm{~dB}(\mathrm{~A})$, resultando em uma relação S/R igual a zero, para obtenção do IPRSR.

Antes da realização dos testes, tanto no silêncio quanto no ruído, foram apresentadas as sentenças iniciais da lista $1 \mathrm{~A}$, para que os indivíduos pudessem compreender a tarefa requerida.

Para a obtenção dos limiares, a técnica de apresentação das sentenças foi baseada na estratégia sequencial, adaptativa ou ascendente-descendente ${ }^{(12)}$, que permite determinar o limiar de reconhecimento de fala, que é o nível necessário para o indivíduo identificar, de forma correta, aproximadamente 50\% dos estímulos de fala apresentados.

O procedimento para a obtenção dos limiares consistiu na apresentação de um estímulo em uma determinada condição, sem ou com ruído competitivo. Se o indivíduo fosse capaz de reconhecer corretamente o estímulo de fala apresentado, a intensidade do mesmo era diminuída em intervalos préestabelecidos. Caso contrário, sua intensidade era aumentada. Esse procedimento foi repetido até o final da lista.

Conforme a literatura, foram utilizados intervalos de $4 \mathrm{~dB}$ até a primeira mudança no tipo de resposta e, posteriormente, os intervalos de apresentação dos estímulos foram de $2 \mathrm{~dB}$ entre si até o final da lista ${ }^{(12)}$.

Para a determinação dos limiares, foi calculada a média dos valores a partir da intensidade de apresentação das sentenças em que ocorreu a primeira mudança de resposta; ou seja, a partir da intensidade da primeira sentença repetida de forma incorreta.

Para a obtenção dos índices percentuais de reconhecimento de sentenças, a intensidade foi mantida fixa durante toda a lista. Para a determinação dos índices, foram somadas todas as sentenças respondidas de forma correta, correspondendo a dez pontos percentuais para cada sentença da lista.

As medidas, realizadas em campo livre, foram efetuadas após a devida calibração do equipamento, tendo em vista as características do sinal de teste e as condições acústicas do ambiente. Nessa calibração, realizada semanalmente, foi estabelecido o nível de pressão sonora no qual o indivíduo testado percebia a fala e o ruído. Para isso, foi utilizado um medidor de pressão sonora digital, da marca Radio Shack®, que foi posicionado em um ponto médio entre as duas orelhas, a uma distância de um metro do alto-falante. A escala de medição utilizada foi a escala $\mathrm{A}^{(7)}$, por ser adequada para mensurar ruídos contínuos e para determinar valores extremos de ruídos intermitentes.
A intensidade de apresentação das sentenças foi calibrada a partir de um tom puro registrado no canal do $\mathrm{CD}$ em que estão gravadas as sentenças. Esse tom puro, um som contínuo de referência, foi utilizado para que as mesmas condições de apresentação fossem sempre mantidas. Isso se deve em virtude de o sinal de fala ser um som complexo, que apresenta uma variação de $30 \mathrm{~dB}$ entre o som mais intenso e o menos intenso, oscilando $12 \mathrm{~dB}$ acima e $18 \mathrm{~dB}$ abaixo da média ${ }^{(13)}$, necessitando, portanto, desse som referencial.

Antes do início das avaliações, a saída de cada canal do CD foi calibrada através do $V U$-meter do audiômetro. Tanto o tom de $1 \mathrm{kHz}$, presente em um canal, quanto o ruído mascarante, presente no outro canal, foram colocados no nível zero.

Estudos realizados anteriormente observaram que as sentenças foram gravadas no $\mathrm{CD}$ em uma intensidade média 7 $\mathrm{dB}$ abaixo da intensidade do tom puro ${ }^{(14)}$. Essa diferença foi levada em consideração e corrigida no dial do equipamento no momento da aplicação dos testes.

As medidas da pesquisa foram obtidas em cabine tratada acusticamente, por meio de um audiômetro digital de dois canais, marca Damplex ${ }^{\circledR}$, modelo DA65; e um sistema de amplificação para audiometria em campo livre, modelo TA 1010. As sentenças foram apresentadas utilizando-se um CD player da marca Britânia ${ }^{\circledR}$, modelo B5279, na opção lineout, acoplado ao audiômetro descrito.

Os dados coletados foram submetidos a uma análise descritiva e a tratamento estatístico por meio da apreciação do comportamento das variáveis, com comparações, para cada grupo, entre os resultados das avaliações efetuadas nos dois diferentes períodos. Para tal, foi utilizado o teste de Wilcoxon, que não exige que os dados sejam provenientes de uma distribuição normal e que testou se os dois valores relacionados apresentaram diferenças estatísticas.

Foi analisado, também, se para cada avaliação executada, ocorreu diferença entre os grupos. Essa comparação foi efetuada com o uso do teste de Mann-Whitney, que testou se os dois valores independentes, e sem distribuição normal, apresentaram diferenças estatisticamente significativas. Para ambos os testes, foi estabelecido um nível de significância de 5\%.

\section{RESULTADOS}

A Tabela 1 ilustra os resultados obtidos nas avaliações do LRSS e LRSR para os grupos Linear e Não Linear e a comparação dos valores encontrados após 14 e 90 dias de uso das próteses auditivas para cada grupo. Os valores encontrados, para ambos os grupos, tanto no silêncio quanto na presença de ruído competitivo, evidenciaram que não houve diferenças.

A Tabela 2 faz uma análise referente somente aos indivíduos que apresentaram melhora nos resultados do LRSS e LRSR, com ruído fixo a $65 \mathrm{~dB}(\mathrm{~A})$, para os grupos estudados. Foi constatado que, para o Grupo Linear, houve melhora em $61,24 \%$ e $30,77 \%$ dos sujeitos, respectivamente, para LRSS e LRSR; para o Grupo Não Linear, melhoraram o desempenho na segunda avaliação $45,83 \%$ e $54,17 \%$ dos sujeitos, respectivamente, para LRSS e LRSR.

A Tabela 3 apresenta os resultados obtidos nas avaliações do IPRSS e IPRSR para os grupos Linear e Não Linear e a 
Tabela 1. Medidas descritivas e p-valor do LRSS e do LRSR, com ruído a $65 \mathrm{~dB}$ A, no $14^{\circ}$ e $90^{\circ}$ dias após a adaptação das próteses auditivas para os grupos Linear e Não Linear

\begin{tabular}{|c|c|c|c|c|c|c|c|c|c|c|c|c|}
\hline \multirow[t]{2}{*}{ Avaliações } & \multicolumn{6}{|c|}{ Grupo Linear } & \multicolumn{6}{|c|}{ Grupo Não Linear } \\
\hline & $\mathrm{N}$ & $\begin{array}{l}\text { Média } \\
d B(A)\end{array}$ & $\begin{array}{c}\text { Limite } \\
\text { inferior } \\
\mathrm{dB}(\mathrm{A})\end{array}$ & $\begin{array}{c}\text { Mediana } \\
\mathrm{dB}(\mathrm{A})\end{array}$ & $\begin{array}{c}\text { Limite } \\
\text { superior } \\
\mathrm{dB}(\mathrm{A})\end{array}$ & Valor de $p$ & $\mathrm{~N}$ & $\begin{array}{l}\text { Média } \\
\mathrm{dB}(\mathrm{A})\end{array}$ & $\begin{array}{c}\text { Limite } \\
\text { inferior } \\
\mathrm{dB}(\mathrm{A})\end{array}$ & $\begin{array}{c}\text { Mediana } \\
\mathrm{dB}(\mathrm{A})\end{array}$ & $\begin{array}{c}\text { Limite } \\
\text { superior } \\
\mathrm{dB}(\mathrm{A})\end{array}$ & Valor de $p$ \\
\hline \multicolumn{13}{|l|}{ LRSS } \\
\hline 14 dias & 13 & 50,02 & 34,33 & 51,28 & 76,67 & 0,4216 & 24 & 45,44 & 36,66 & 43,64 & 61,29 & 0,9544 \\
\hline 90 dias & 13 & 49,11 & 32,20 & 53,00 & 69,22 & & 24 & 45,24 & 34,00 & 44,50 & 60,78 & \\
\hline \multicolumn{13}{|l|}{ LRSR } \\
\hline 14 dias & 13 & 64,75 & 60,00 & 63,89 & 76,67 & 0,2942 & 24 & 63,48 & 58,50 & 63,52 & 68,00 & 0,8638 \\
\hline 90 dias & 13 & 65,78 & 61,22 & 64,11 & 75,89 & & 24 & 63,35 & 56,71 & 63,11 & 70,56 & \\
\hline
\end{tabular}

Teste de Wilcoxon $(\mathrm{p} \leq 0,05)$

Legenda: LRSS = limiar de reconhecimento de sentenças no silêncio; LRSR = limiar de reconhecimento de sentença no ruído

Tabela 2. Análise referente aos indivíduos que apresentaram melhora nas avaliações do LRSS e LRSR para os grupos Linear e Não Linear

\begin{tabular}{llccccc}
\hline Avaliações & \multicolumn{4}{c}{ Grupo Linear } & \multicolumn{3}{c}{ Grupo Não Linear } \\
\cline { 2 - 7 } & $\mathrm{N}$ & $\%$ & Média da melhora dB(A) & $\mathrm{N}$ & $\%$ & Média da melhora dB(A) \\
\hline LRSS & 8 & 61,24 & 4,32 & 11 & 45,83 & 3,46 \\
LRSR & 4 & 30,77 & 1,57 & 13 & 54,17 & 2,24 \\
\hline
\end{tabular}

Legenda: LRSS = limiar de reconhecimento de sentenças no silêncio; LRSR = limiar de reconhecimento de sentença no ruído

Tabela 3. Medidas descritivas e p-valor do IPRSS e do IPRSR, com ruído a 65 dB A, no $14^{\circ}$ e $90^{\circ}$ dias após a adaptação das próteses auditivas para os grupos Linear e Não Linear

\begin{tabular}{|c|c|c|c|c|c|c|c|c|c|c|c|c|}
\hline \multirow[t]{2}{*}{ Avaliações } & \multicolumn{6}{|c|}{ Grupo Linear } & \multicolumn{6}{|c|}{ Grupo Não Linear } \\
\hline & $\mathrm{N}$ & $\begin{array}{l}\text { Média } \\
\mathrm{dB}(\mathrm{A})\end{array}$ & $\begin{array}{c}\text { Limite } \\
\text { inferior } \\
\mathrm{dB}(\mathrm{A})\end{array}$ & $\begin{array}{c}\text { Mediana } \\
\mathrm{dB}(\mathrm{A})\end{array}$ & $\begin{array}{c}\text { Limite } \\
\text { superior } \\
\mathrm{dB}(\mathrm{A})\end{array}$ & Valor de $p$ & $\mathrm{~N}$ & $\begin{array}{l}\text { Média } \\
\mathrm{dB}(\mathrm{A})\end{array}$ & $\begin{array}{l}\text { Limite } \\
\text { inferior } \\
\mathrm{dB}(\mathrm{A})\end{array}$ & $\begin{array}{c}\text { Mediana } \\
\mathrm{dB}(\mathrm{A})\end{array}$ & $\begin{array}{c}\text { Limite } \\
\text { superior } \\
\mathrm{dB}(\mathrm{A})\end{array}$ & Valor de $p$ \\
\hline \multicolumn{13}{|l|}{ IPRSS } \\
\hline 14 dias & 13 & 93,07 & 10 & 100 & 100 & 0,3173 & 24 & 96,67 & 50 & 100 & 100 & 0,0835 \\
\hline 90 dias & 13 & 90,00 & 10 & 100 & 100 & & 24 & 100 & 100 & 100 & 100 & \\
\hline \multicolumn{13}{|l|}{ IPRSR } \\
\hline 14 dias & 13 & 61,53 & 10 & 60 & 100 & 0,6972 & 24 & 73,75 & 00 & 80 & 100 & 0,7269 \\
\hline 90 dias & 13 & 63,07 & 00 & 70 & 100 & & 24 & 75,00 & 30 & 80 & 100 & \\
\hline
\end{tabular}

Teste de Wilcoxon $(p \leq 0,05)$

Legenda: IPRSS = índice percentual de reconhecimento de sentenças no silêncio; IPRSR = índice percentual de reconhecimento de sentenças no ruído

Tabela 4. Análise referente aos indivíduos que apresentaram melhora nas avaliações do IPRSR para os grupos Linear e Não Linear

\begin{tabular}{lcccccc}
\hline Avaliação & \multicolumn{3}{c}{ Grupo Linear } & & \multicolumn{2}{c}{ Grupo Não Linear } \\
\cline { 2 - 6 } & $\mathrm{N}$ & $\%$ & $\begin{array}{c}\text { Média da } \\
\text { melhora (\%) }\end{array}$ & & N & $\begin{array}{c}\text { Média da } \\
\text { melhora (\%) }\end{array}$ \\
\hline IPRSR & 6 & 46,15 & 20 & 9 & 37,50 & 21 \\
\hline
\end{tabular}

Legenda: IPRSR = índice percentual de reconhecimento de sentenças no ruído

comparação dos valores encontrados após 14 e 90 dias de uso das próteses auditivas para cada grupo. Quando comparados os resultados das avaliações realizadas, não foram encontradas diferenças em nenhum dos grupos.

A Tabela 4 faz uma análise referente somente aos indivíduos que apresentaram melhora nos resultados do IPRSS e IPRSR, com ruído fixo a $65 \mathrm{~dB}(\mathrm{~A})$, para os grupos estudados. Foi verificado que $46,15 \%$ dos sujeitos do Grupo Linear e $37,50 \%$ dos componentes do Grupo Não Linear melhoraram os desempenhos no IPRSR, com modificações médias de $20 \%$ e $21 \%$, respectivamente.

A Tabela 5 apresenta, para todas as variáveis pesquisadas, informações sobre a diferença entre os valores encontrados para usuários de amplificação linear e aqueles obtidos por usuários de amplificação não linear. As comparações entre os grupos apontaram para a inexistência de diferenças.

\section{DISCUSSÃO}

Os valores encontrados na pesquisa dos limiares, ilustrados na Tabela 1, apontaram que, tanto para os indivíduos do Grupo Linear quanto para os sujeitos do Grupo Não Linear, não foram evidenciadas diferenças estatísticas na comparação entre as avaliações realizadas após 14 e 90 dias da adaptação de próteses 
Tabela 5. Valores de p na comparação entre os grupos Linear e Não Linear para LRSS, IPRSS, LRSR e IPRSR no $14^{\circ}$ e $90^{\circ}$ dias após a adaptação das próteses auditivas

\begin{tabular}{lcc}
\hline & Variáveis & $\begin{array}{c}\text { Grupo Linear } x \text { Grupo Não Linear } \\
\text { Valor de } p\end{array}$ \\
\hline $14^{\circ}$ dia & LRSS & 0,3162 \\
& LRSR & 0,5139 \\
& IPRSS & 0,7233 \\
& IPRSR & 0,0671 \\
\hline $90^{\circ}$ dia & LRSS & 0,2654 \\
& LRSR & 0,0694 \\
& IPRSS & 0,0514 \\
& IPRSR & 0,2953 \\
\hline
\end{tabular}

Teste de Mann-Whitney $(p \leq 0,05)$

Legenda: LRSS = limiar de reconhecimento de sentenças no silêncio; LRSR = limiar de reconhecimento de sentença no ruído; IPRSS = índice percentual de reconhecimento de sentenças no silêncio; IPRSR = índice percentual de reconhecimento de sentenças no ruído

auditivas. De maneira geral, podemos inferir que não ocorreu modificação no reconhecimento de fala em nenhum dos grupos entre o $14^{\circ}$ e o $90^{\circ}$ dia posterior ao início do uso da amplificação.

Embora nossos achados, de forma geral, não tenham sido condizentes com os efeitos da aclimatização, concordamos que mudanças são perceptíveis ao longo do tempo, mesmo que haja um evidente conflito relativo à existência da aclimatização, uma vez que algumas pesquisas confirmaram sua ocorrência, enquanto outras fracassaram na tentativa de demonstrar seus efeitos ${ }^{(15)}$. Existem, no mínimo, três possíveis explicações para justificar os estudos que não foram capazes de demonstrar a ocorrência da aclimatização: sujeitos com poucas oportunidades de melhora (uso limitado das próteses auditivas), emprego de testes não sensíveis às modificações que ocorreram e/ou testes utilizados de forma errônea para tentar demonstrar a aclimatização ${ }^{(15)}$. No presente estudo, consideramos a hipótese que boa parte dos pacientes participantes pode ter efetuado o uso das próteses de forma limitada durante os primeiros meses seguintes à adaptação, apesar de ter recebido as devidas orientações e ter acesso a consultas fonoaudiológicas.

Conforme a literatura, os efeitos da aclimatização estão na dependência do tipo de amplificação e da experiência prévia com amplificação. Foi realizada uma pesquisa que acompanhou os efeitos da aclimatização em novos usuários de próteses auditivas, adaptados bilateralmente com amplificação linear ou com amplificação não linear do tipo WDRMCC (Wide Dynamic Range Multichannel Compression). O estudo analisou dois grupos de indivíduos que iniciaram o uso de próteses com um dos tipos de amplificação e, depois de 32 semanas, tiveram o tipo de amplificação substituído entre os grupos. Foram verificadas claras evidências dos efeitos da aclimatização nos novos usuários de próteses auditivas com processamento WDRMCC. Os novos usuários adaptados com amplificação linear não apresentaram resultados consistentes com os efeitos da aclimatização. Após a troca entre os tipos de amplificação, foram percebidas pequenas evidências da aclimatização, tanto para o grupo que substituiu WDRMCC por linear quanto para o grupo que substituiu linear por WDRMCC. Assim, nenhum dos efeitos da aclimatização pôde ser comparado à magnitude inicial alcançada com o uso do processamento WDRMCC ${ }^{(16)}$.

Em nosso estudo, quando realizamos uma análise individual, com o intuito de verificar somente aqueles indivíduos que apresentaram resultados melhores na segunda avaliação, conforme descrito na Tabela 2, foi possível verificar que, dentre os integrantes do Grupo Linear, 61,24\% dos sujeitos melhoraram o desempenho no LRSS em 4,32 dB, em média; e 30,77\% dos sujeitos aprimoraram o resultado para o LRSR em 1,57 dB, em média. Já para os componentes do Grupo Não Linear, a mesma análise individual assinalou que 45,83\% melhoraram o desempenho no LRSS em 3,46 dB, em média; e 54,17\% obtiveram resultados melhores para o LRSR em 2,24 dB, em média.

Desta forma, foi observado que, para os grupos estudados, independente do tipo de amplificação utilizada pelo portador de perda auditiva, para um considerável número de sujeitos ocorreram indícios de plasticidade neural e efeitos da aclimatização; entretanto, verificou-se que, em ambos os grupos, muitos indivíduos não demonstraram quaisquer modificações no benefício obtido com o uso das próteses auditivas, e determinados pacientes até pioraram seus desempenhos.

Atribuímos esse episódio a fatores particulares, uma vez que o benefício depende do ambiente acústico em que cada usuário de próteses está inserido e é significativamente influenciado por características individuais ${ }^{(17)}$.

Com relação aos índices percentuais de reconhecimento de sentenças, não foram encontradas diferenças estatísticas em nenhum dos grupos, quando comparados os resultados das avaliações realizadas no $14^{\circ}$ e no $90^{\circ}$ dias subsequentes à adaptação das próteses auditivas (Tabela 3 ).

A obtenção do IPRSS foi realizada com os estímulos de fala fixos na intensidade de $65 \mathrm{~dB}(\mathrm{~A})$. Ao analisar os resultados, constatamos, para ambos os grupos, que quase todos os indivíduos apresentaram desempenho igual a $100 \%$ na primeira avaliação. Verificamos, com isso, que esse procedimento não foi apropriado para examinar a influência do tempo de uso da amplificação sobre o benefício obtido com as próteses, uma vez que, da forma empregada, não permitiu que os indivíduos fossem capazes de atingir melhores resultados na segunda avaliação. Indicamos, para trabalhos futuros que tenham o intuito de obter IPRSS, que o valor determinante no qual o nível de intensidade deva ser fixado seja realizado com base no resultado individual obtido na avaliação do LRSS.

Para a determinação do IPRSR, adotamos fala e ruído a 65 $\mathrm{dB}(\mathrm{A})$, resultando em uma relação $\mathrm{S} / \mathrm{R}$ fixa igual a zero. Em análise, não ocorreu, de forma geral, melhora no reconhecimento de fala pelos indivíduos do Grupo Linear nem dos indivíduos do Grupo Não Linear, no período compreendido entre 14 e 90 dias de uso da amplificação. No entanto, ao efetuarmos um julgamento por indivíduo, com o propósito de verificar somente aqueles que apresentaram resultados melhores na segunda avaliação, foi possível conferir que, para o IPRSR, 46,15\% dos sujeitos integrantes do Grupo Linear apresentaram modificações médias de $20 \%$ para melhor em seus resultados; e 37,50\% dos componentes do Grupo Não Linear tiveram seu desempenho melhorado em $21 \%$, em média (Tabela 4).

Essas diferenças individuais encontradas podem ser decorrentes de aspectos de personalidade, motivação e expectativa 
frente às próteses ${ }^{(17)}$. Associado a isso, há relatos na literatura no sentido de que seria necessário, a fim de examinar os efeitos da aclimatização, que as avaliações de benefício também fossem realizadas com o uso de medidas subjetivas e mensurações eletrofisiológicas ${ }^{(1)}$. Cabe-nos acrescentar, ainda, que nenhum de nossos pacientes foi submetido a treinamento auditivo e apontar, desse modo, que somente para parte dos sujeitos desta pesquisa, as habilidades de reconhecimento de fala melhoraram espontaneamente ao longo do tempo. Assim, podemos considerar que o treinamento auditivo seria uma grande ajuda para todos os indivíduos que recebem adaptação de próteses auditivas, em especial, aqueles em que modificações espontâneas não são observadas no decorrer do tempo.

Nosso estudo também investigou se, para todas as avaliações e reavaliações efetuadas, existiriam diferenças nos resultados apresentados pelos pacientes que receberam adaptação de próteses auditivas com amplificação linear dos escores obtidos pelos pacientes que receberam adaptação de próteses com compressão dinâmica -WDRC. As comparações realizadas entre os grupos, para todas as variáveis pesquisadas, estão demonstradas na Tabela 5 (Grupo Linear versus Grupo Não Linear).

O teste estatístico empregado indicou não haver diferenças nas comparações entre os grupos. Esses achados não estão de acordo com um trabalho realizado no ano de $2002^{(18)}$, em que foi encontrada diferença estatística entre diferentes circuitos de próteses auditivas; entretanto, corroboram outros estudos recentes ${ }^{(2,19)}$, em que também não foram encontradas diferenças estatísticas na comparação entre próteses auditivas lineares e não lineares.

Pesquisa realizada em 2002 comparou o desempenho no reconhecimento de fala para três circuitos de próteses auditivas: cortes de pico, compressão por limitação e WDRC. Foram examinados 360 indivíduos, divididos em quatro grupos de acordo com o grau e a configuração da perda auditiva. Diferenças significativas favoreceram os circuitos corte de pico e compressão por limitação sobre o circuito WDRC nos grupos com perda auditiva de grau leve; e favoreceram o WDRC sobre o corte de pico nos grupos com perda auditiva de grau superior ao leve $\mathrm{e}^{(18)}$.

Em 2004 foi realizado um estudo comparando os benefícios, em indivíduos idosos, com o uso de próteses auditivas lineares e próteses com processamento WDRC, com adapta- ção binaural. Os exames indicaram que ambas as tecnologias proporcionaram desempenho satisfatório para seus usuários, não havendo diferença entre amplificação linear e WDRC ${ }^{(19)}$.

Em outro estudo ${ }^{(2)}$, que objetivou comparar os resultados obtidos e verificar o circuito que favoreceu melhor adaptação auditiva e reconhecimento de fala entre usuários de próteses auditivas com circuitos linear e não linear, foi constatado não haver diferenças no reconhecimento de fala, tanto no silêncio quanto no ruído. Embora os resultados não tenham apresentado diferenças significativas, as autoras reportaram que, para o LRSS, os indivíduos usuários de amplificação não linear apresentaram melhores resultados; já para o LRSR, os resultados foram semelhantes. Em nosso estudo, também foi possível verificar resultados melhores a favor do Grupo Não Linear, porém esses resultados favoráveis foram encontrados em todas as variáveis pesquisadas.

Assim, foi possível apurar que houve uma tendência de os indivíduos do Grupo Não Linear apresentar melhores resultados, tanto para situações de silêncio quanto na presença de ruído competitivo. Apesar disso, reiteramos que as diferenças verificadas não foram significativas.

Com base em nossos achados, acreditamos que as próteses auditivas devam contemplar seus usuários com qualidade sonora e, principalmente, satisfação. O fonoaudiólogo deve, no processo de seleção e adaptação de próteses auditivas, considerar possíveis benefícios decorrentes do uso da amplificação, adequar a tecnologia às necessidades e entender que todo esse processo precisa ser realizado de maneira personalizada. Deve, ainda, valorizar o uso de avaliações subjetivas, com a aplicação de questionários de auto-avaliação, como um instrumento de auxílio para todo esse processo.

\section{CONCLUSÃO}

Não foram verificadas, por meio do teste LSP, influências do tempo de uso da amplificação sobre o benefício obtido com as próteses auditivas para novos usuários de amplificação linear e novos usuários de amplificação não linear.

Não existiram diferenças nos resultados alcançados pelos novos usuários de amplificação linear daqueles obtidos pelos novos usuários de amplificação não linear.

\title{
ABSTRACT
}

\begin{abstract}
Purpose: To verify the influence of the period of amplification use over the benefit obtained with hearing aids for new users, and to compare the results of users of linear and non linear amplification with dynamic compression. Methods: Thirty-seven individuals with mild to moderate-severe sensorineural hearing loss who were new users of hearing aids were examined. Individuals were gathered in two groups: Linear Group - comprising 13 individuals with linear amplification fitting; and Non Linear Group - comprising 24 individuals with dynamic compression fitting. Evaluations were carried out 14 and 90 days after hearing aids fitting, in sound field. The sentence recognition threshold was obtained both in silence and in noise, as well as the percentual indexes of sentences recognition. Data were analyzed using the Wilcoxon and the Mann-Whitney tests. Results: The values obtained after 14 and 90 days of amplification use, for both groups, did not show significant differences. The values also did not reveal any differences when the groups were compared. Conclusion: It was not found any influences of the period of amplification use over the benefit obtained from hearing aids. There were no differences between the results achieved by new users of linear amplification and those of new users of non linear amplification.
\end{abstract}

Keywords: Hearing; Hearing loss, sensorineural; Hearing aids; Audiometry, speech; Speech discrimination tests 


\section{REFERENCIAS}

1. Amorim RMC, Almeida K. Estudo do benefício e da aclimatização em novos usuários de próteses auditivas. Pró-Fono. 2007;19(1):39-48.

2. Costa LP, Iório MCM. Próteses auditivas: avaliações objetivas e subjetivas em usuários de amplificação linear e não-linear. Pró-Fono. 2006;18(1):21-30.

3. Bucuvic EC, Iório MCM. Próteses auditivas: estudo comparativo das dificuldades auditivas e do benefício da amplificação em pacientes usuários de amplificação não linear e linear. Rev Ciên Med Biol. 2003;2(1):77-87.

4. Menegotto IH, Iório MCM. Processamento do sinal sonoro nas próteses auditivas: compressão. In: Almeida K, Iório MCM. Próteses auditivas: fundamentos teóricos e aplicações clínicas. 2a ed. São Paulo: Lovise; c2003. p. 119-48.

5. Gatehouse S. The time course and magnitude of perceptual acclimatization to frequency responses: evidence from monoaural fitting of hearing aids. J Acoust Soc Am. 1992;92(3):1258-68.

6. Reber MB, Kompis M. Acclimatization in first-time hearing aid users using three different fitting protocols. Auris Nasus Larynx. 2005;32(4):345-51.

7. Costa MJ. Listas de sentenças em português: apresentação e estratégias de aplicação na audiologia. Santa Maria: Gráfica e Editora Pallotti; 1998.

8. Silman S, Silverman CA. Auditory diagnosis: principles and applications. London: Singular Publishing Group; 1991.

9. Costa MJ, Iório MCM, Mangabeira-Albernaz PL. Reconhecimento de fala: desenvolvimento de uma lista de sentenças em português. Acta AWHO. 1997;16(4):164-73.

10. Costa MJ. Desenvolvimento de listas de sentenças em português [tese]. São Paulo: Universidade Federal de São Paulo. Escola Paulista de Medicina; 1997.
11. Costa MJ, Iorio MCM, Albernaz PLM, Cabral Júnior EF, Magni AB. Desenvolvimento de um ruído com espectro de fala. Acta AWHO. 1998;17(2):84-9.

12. Levitt H, Rabiner LR. Use of a sequencial strategy in intelligibility testing. J Acoust Soc Am. 1967;42(3):609-12.

13. Boothroyd A. Speech perception, sensorioneural hearing loss and hearing aids. In: Studebaker GA, Hochberg I. Acoustical factors affecting hearing aid performance. 2nd ed. Boston: Allyn \& Bacon; 1992. p. 277-99.

14. Cóser PL, Costa MJ, Cóser MJS, Fukuda Y. Reconhecimento de sentenças no silêncio e no ruído em indivíduos portadores de perda auditiva induzida pelo ruído. Rev Bras Otorrinolaringol. 2000;66(4):362-70.

15. Munro KJ, Lutman ME. The effect of speech presentation level on measurement of auditory acclimatization to amplified speech. J Acoust Soc Am. 2003;114(1):484-95.

16. Yund EW, Roup CM, Simon HJ, Bowman GA. Acclimatization in wide dynamic range multichannel compression and linear amplification hearing aids. J Rehabil Res Dev. 2006;43(4):517-36.

17. Gatehouse S, Naylor G, Elberling C. Benefits from hearing aids in relation to the interaction between the user and the environment. Int $\mathrm{J}$ Audiol. 2003;42 Suppl 1: S77-85.

18. Shanks JE, Wilson RH, Larson V, Williams D. Speech recognition performance of patients with sensorineural hearing loss under unaided and aided conditions using linear and compression hearing AIDS. Ear Hear. 2002;23(4):280-90.

19. Humes LE, Humes LE, Wilson DL. A comparison of single-channel linear amplification and two-channel wide-dynamic-range-compression amplification by means of an independent-group design. Am J Audiol. 2004;13(1):39-53. 